\title{
Allozyme Genetics in Permanent Translocation Heterozygotes of the Oenothera biennis Complex
}

\author{
Morris Levy, ${ }^{1,2}$ Erich E. Steiner, ${ }^{3}$ and Donald A. Levin ${ }^{1}$
}

Received 17 Dec. 1974-Final 21 Feb. 1975

Allozyme inheritance and transmission genetics of 11 enzyme systems were determined in the permanent translocation heterozygotes Oenothera biennis, Oe. strigosa, and Oe. parviflora. Electrophoretic variation was examined first among 164 strains of structural heterozygotes. Allelic configurations were then judged from inheritance patterns in reciprocal $F_{1}$ hybrids between each of 22 ring-forming strains and tester strains of the related bivalent-formers, Oe. hookeri and Oe. grandiffora. Allozymes are inherited as codominant markers, and, as dictated by the genetic system, within a strain individual allelic variants are generally transmitted through only one germ line. Of the 20 loci resolved, only eight are polymorphic in any species, and, within species, generally only two alleles are present at each polymorphic locus. Despite the relatively meager allelic array, each of the 22 strains whose chromosome complexes were characterized is genotypically unique. Generally, within taxa, $\alpha$ (egg) and $\beta$ (sperm) complexes differ in allele frequency at several polymorphic loci. Such variability is correlated with differences in the phylogenetic origins of complexes and not with differences in segmental arrangement within a group of related complexes.

KEY WORDS: allozyme genetics; Oenothera; translocation heterozygosity.

\section{INTRODUCTION}

The cytogenetic studies of Cleland and associates ( $c f$. Cleland, 1972) in Oenothera have provided insight into the phylogeny and the evolution of complete and permanent translocation heterozygosity in certain taxa of the

The study was supported by NIH Training Grant in Genetics 5 TO 1 GM-00337-13.

1 Department of Botany, University of Texas, Austin, Texas.

2 Present address: Department of Biological Sciences, Purdue University, West Lafayette, Indiana.

${ }^{3}$ Department of Botany, University of Michigan, Ann Arbor, Michigan.

(C) 1975 Plenum Publishing Corporation, 227 West 17th Street, New York, N.Y. 10011. No part of this publication may be reproduced, stored in a retrieval system, or transmitted, in any form or by any means, electronic, mechanical, photocopying, microfilming, recording, or otherwise, without written permission of the publisher. 
genus. However, investigations of population dynamics and ecological genetics have been hampered by the lack of single locus markers which can be readily identified. With the development of gel electrophoresis and substrate-specific straining recipes, allozyme variants may be utilized as such markers, thus permitting rapid analyses of genetic variation and heterozygosity in populations and population systems. The present study focuses on the genetic bases of electrophoretic variation in the permanent translocation heterozygotes of the North American subgenus Euoenothera, Oe. biennis, $O e$. strigosa, and Oe. parviftora. Companion studies utilizing allozyme variation to investigate geographical variation, phylogenetic affinity, and population structure in these taxa and related bivalent-forming oenotheras appear elsewhere (Levin et al., 1972; Levy and Levin, 1975; Levin, 1975).

The major features of structural hybridity in the Oenothera biennis complex are as follows ( $c f$. Cleland, 1972). Each structurally heterozygous species contains two seven-chromosome genomes or "Renner" complexes which differ completely in chromosome end (segmental) arrangement. A ring of 14 chromosomes is formed at diakinesis, and, following alternate disjunction, each complex is reconstituted within single gametes. Structural hybridity is maintained by a system of balanced gametophytic and sporophytic lethals; each complex tends to be transmitted exclusively through only one of the germ lines. The genetic system thus insures that strains are truebreeding and that heterozygosity is maintained despite predominant selffertilization.

Egg $(\alpha)$ and sperm $(\beta)$ complexes in each ring-forming taxon uniformly manifest distinct morphological syndromes; ostensibly each taxon is a stabilized hybrid derivative. Oenothera biennis has diverged into three groups, Oe. biennis $I$ and Oe. biennis $I I$, which contain a "biennis" and a "strigosa" genome, and Oe. biennis III, which contains two "biennis" genomes. Genome transmission in Oe. biennis $I$ and Oe. biennis II is reversed, the "biennis" genome residing in the $\alpha$ complex of Oe. biennis $I$ and in the $\beta$ complex of $O e$. biennis II. Ancestral sources of $\alpha$ and $\beta$ complexes in the other ringforming taxa are not as well defined. Oenothera strigosa contains two very similar "strigosa" genomes. Both groups of Oe. parviflora contain highly disparate genomes. Morphological syndromes of the $\beta$ complexes of both groups resemble the relict Oe. argillicola; $\alpha$-parviftora I complexes are "biennis"-like, and $\alpha$-parviflora II complexes manifest "strigosa" morphology.

\section{MATERIALS AND METHODS}

Samples of geographically widespread strains, cytogenetically characterized by Cleland and others, were obtained from the U.S. National Seed Storage Laboratory, Fort Collins, Colorado. These included representatives of Oe. 
biennis I (57), Oe. biennis II (43), Oe. biennis III (6), Oe. strigosa (29), Oe. parviflora I (19), and Oe. parviflora II (10). Individual 2-week-old seedlings of each strain were assayed electrophoretically for acid phosphatase (ACP), alkaline phosphatase (ALP), glucose 6-phosphate dehydrogenase (G6PD), glutamate oxalate transaminase (GOT), leucine aminopeptidase (LAP), leucyl-glycyl-glycine peptidase (LGGP), leucyl-tyrosine peptidase (LTP), malate dehydrogenase (MDH), phosphoglucose isomerase (PGI), phosphoglucose mutase (PGM), and valyl-leucine peptidase (VLP) following procedures detailed elsewhere (Levy and Levin, 1975).

A total of 22 ring-forming strains, collectively representing nearly all electrophoretic variation detected in the survey, were crossed both as male parent and as female parent to three bivalent-forming tester strains, two of Oe. hookeri and one of Oe. grandiflora. Three tester strains were used to anticipate potential crossing or $F_{1}$ germination difficulties and for independent confirmations of allozyme transmission genetics. Twenty-one of the 22 ring-forming strains were polymorphic for one or more enzyme systems, and each of the tester strains had been inbred for many generations and was monomorphic for all systems. Ostensibly, $F_{1}$ hybrids would contain only the $\alpha$ or $\beta$ complex of a structural heterozygote and, consequently, only those allozymes specific to that complex. Allozyme genetics were judged from inheritance patterns of electrophoretic phenotypes in the reciprocal $F_{1}$ hybrids. Fourteen seedlings in each hybrid line were analyzed for each enzyme system.

\section{RESULTS AND DISCUSSION}

\section{Electrophoretic Variation}

Electrophoretic banding patterns manifest in each taxon as characterized by migration distance relative to the anodal electrophoretic front $\left(R_{p}\right)$ are summarized in Table I. All resolved systems migrated anodally. Multiple zones of staining, distinctive in migration distance and between which no segregation was observed, were present for ACP, ALP, GOT, LGGP, LTP, PGI, and PGM in all taxa. For these enzyme systems, each zone was numbered progressively from least to greatest mobility from the origin. Collectively, 20 staining zones were resolved among the 11 enzymes analyzed. In 18 zones, electrophoretic phenotypes consisted of either single bands or complementary doublets [two bands with equal staining intensity accompanied by a faintly staining band(s) of intermediate mobility]. For G6PD a doublet was present in all strains, and in GOT-1 only doublets or complementary quadruplets were observed.

All taxa exhibited a common and invariant phenotype for ACP-1, ACP-2, ALP-1, ALP-3, G6PD, LGGP-1, LGGP-2, LTP-1, and MDH. 
Table I. Electrophoretic Variation in Permanent Translocation Heterozygotes and Tester Bivalent-Forming Strains of Oenothera

\begin{tabular}{|c|c|c|c|c|c|}
\hline \multirow{2}{*}{$\begin{array}{l}\text { Enzyme- } \\
\text { staining } \\
\text { zone }\end{array}$} & \multicolumn{5}{|c|}{ Banding phenotypes $\left(R_{p} \times 100\right)$} \\
\hline & biennis & strigosa & parviflora & hookeri & grandiflora \\
\hline ACP-1 & 27 & 27 & 27 & 27 & 27 \\
\hline ACP-2 & 61 & 61 & 61 & 61 & 61 \\
\hline ALP-1 & 30 & 30 & 30 & 30 & 30 \\
\hline \multirow[t]{2}{*}{ ALP-2 } & 60 & 60 & - & 60 & 60 \\
\hline & - & - & 67 & - & - \\
\hline ALP-3 & 93 & 93 & 93 & 93 & 93 \\
\hline G6PD & $44 / 49$ & $44 / 49$ & $44 / 49$ & $44 / 49$ & $44 / 49$ \\
\hline \multirow[t]{6}{*}{ GOT-1 } & $40 / 49$ & $40 / 49$ & - & $40 / 49$ & - \\
\hline & $40 / 45 / 49 / 51$ & $40 / 45 / 49 / 51$ & - & - & -- \\
\hline & $45 / 51$ & - & - & - & $45 / 51$ \\
\hline & - & - & $38 / 46$ & - & - \\
\hline & 一 & - & $38 / 43 / 46 / 48$ & - & $\div$ \\
\hline & 二 & $-\square$ & $43 / 48$ & - & - \\
\hline \multirow[t]{2}{*}{ GOT-2 } & 57 & 57 & - & 57 & 57 \\
\hline & - & $\bar{c}$ & 59 & $\overline{c 5}$ & $\overrightarrow{65}$ \\
\hline \multirow[t]{3}{*}{ LAP } & 65 & 65 & - & 65 & 65 \\
\hline & 一 & - & $60 / 62$ & - & - \\
\hline & - & - & 62 & - & - \\
\hline LGGP-1 & 50 & 50 & $\begin{array}{c}62 / 64 \\
50\end{array}$ & $\overline{50}$ & $\overline{50}$ \\
\hline LGGP-2 & 65 & 65 & 65 & 65 & 65 \\
\hline LTP-1 & 33 & 33 & 33 & 33 & 33 \\
\hline \multirow[t]{4}{*}{ LTP-2 } & $55 / 60$ & - & - & - & - \\
\hline & 60 & 60 & - & 60 & 60 \\
\hline & - & 一 & $56 / 61$ & - & - \\
\hline & - & - & 61 & - & - \\
\hline \multirow[t]{3}{*}{ LTP-3 } & 77 & 77 & - & 77 & 77 \\
\hline & - & - & $74 / 79$ & - & - \\
\hline & 一 & - & 79 & - & - \\
\hline $\mathrm{MDH}$ & 88 & 88 & 88 & 88 & 88 \\
\hline \multirow[t]{5}{*}{ PGI-1 } & $22 / 25$ & $22 / 25$ & - & - & - \\
\hline & 25 & 25 & - & 25 & 25 \\
\hline & 一 & - & 16 & - & - \\
\hline & 一 & - & $16 / 26$ & - & - \\
\hline & $一$ & - & 26 & - & - \\
\hline \multirow[t]{3}{*}{ PGI-2 } & 40 & 40 & 40 & 40 & 40 \\
\hline & 43 & - & - & - & - \\
\hline & $40 / 43$ & $40 / 43$ & $40 / 43$ & - & - \\
\hline \multirow{8}{*}{ PGM-1 } & 38 & - & - & - & - \\
\hline & $38 / 43$ & $38 / 43$ & - & - & - \\
\hline & $\begin{array}{c}43 \\
43 / 47\end{array}$ & 43 & - & $\begin{array}{l}43 \\
-\end{array}$ & 43 \\
\hline & 47 & 47 & - & - & - \\
\hline & - & - & $35 / 39$ & - & - \\
\hline & - & - & $35 / 47$ & - & - \\
\hline & - & - & 39 & - & - \\
\hline & - & $\overline{\sigma x}$ & $39 / 47$ & - & - \\
\hline \multirow[t]{2}{*}{ PGM-2 } & 57 & 57 & - & 57 & 57 \\
\hline & - & $一$ & 55 & - & - \\
\hline
\end{tabular}


Table I. Continued

\begin{tabular}{cccccc}
\hline \multirow{2}{*}{$\begin{array}{c}\text { Enzyme- } \\
\text { staining } \\
\text { zone }\end{array}$} & biennis & strigosa & parviflora & hookeri & grandiflora \\
\cline { 2 - 6 } VLP & 48 & - & - & - & - \\
& $48 / 51$ & $48 / 51$ & - & - & - \\
& $48 / 55$ & $48 / 55$ & - & - & - \\
& 51 & 51 & - & - & - \\
& 55 & 55 & - & 55 & - \\
& - & - & 50 & - & - \\
& - & - & $50 / 51$ & - & - \\
& - & - & $51 / 53$ & - & - \\
\hline
\end{tabular}

Each taxon also exhibited only one phenotype for ALP-2, GOT-2, and PGM2, with Oe parviflora bands being distinctive from those shared by $O e$. biennis and Oe. strigosa. Variation within a taxon was detected in only eight of the 20 staining zones. Each taxon was polymorphic for GOT-1, PGI-1, PGI-2, PGM-1, and VLP. Polymorphism for LTP-2 was detected only in Oe biennis and Oe parviflora and for LTP-3 and LAP only in Oe. parviflora. In polymorphic systems, Oe. biennis and Oe. strigosa exhibited common variants while those of Oe. parviflora were distinctive except for PGI-2. Each tester strain was monomorphic for 18 zones, expressed the usual doublet at G6PD and GOT-1, and exhibited a composite phenotype common only to $O e$. biennis and Oe. strigosa. The Oe. hookeri and Oe. grandiflora testers were distinctive inter se for GOT-1 and VLP (see Table II).

\section{Inheritance Patterns and Allozyme Genotypes}

Eighty-seven hybrid lines, established from 110 attempted crosses, yielded electrophoretic phenotype data. Crossing and germination difficulties were greatest among the tester $\times$ Oe. parviflora crosses. Six of these failed to produce germinable seed and two produced partially or wholly chlorotic seedlings. Such results are consistent with those obtained in other interspecific crosses with $\beta$-parviflora complexes (Cleland, 1972).

A sample of the $F_{1}$ electrophoretic phenotype data, illustrating the spectrum of inheritance patterns observed, is presented in Table II. With certain exceptions, the salient features of the synthetic stocks are that (1) each hybrid line is phenotypically uniform, (2) reciprocal crosses yield different phenotypes for each parental polymorphism, and (3) the phenotype of $F_{1}$ lines is dependent on the direction of the cross. Consider $F_{1}$ phenotype patterns for GOT-1 in crosses involving the Oe. biennis I strain Bloomington $I I$, which exhibits the polymorphic quadruplet 40/45/49/51 (Table II). 


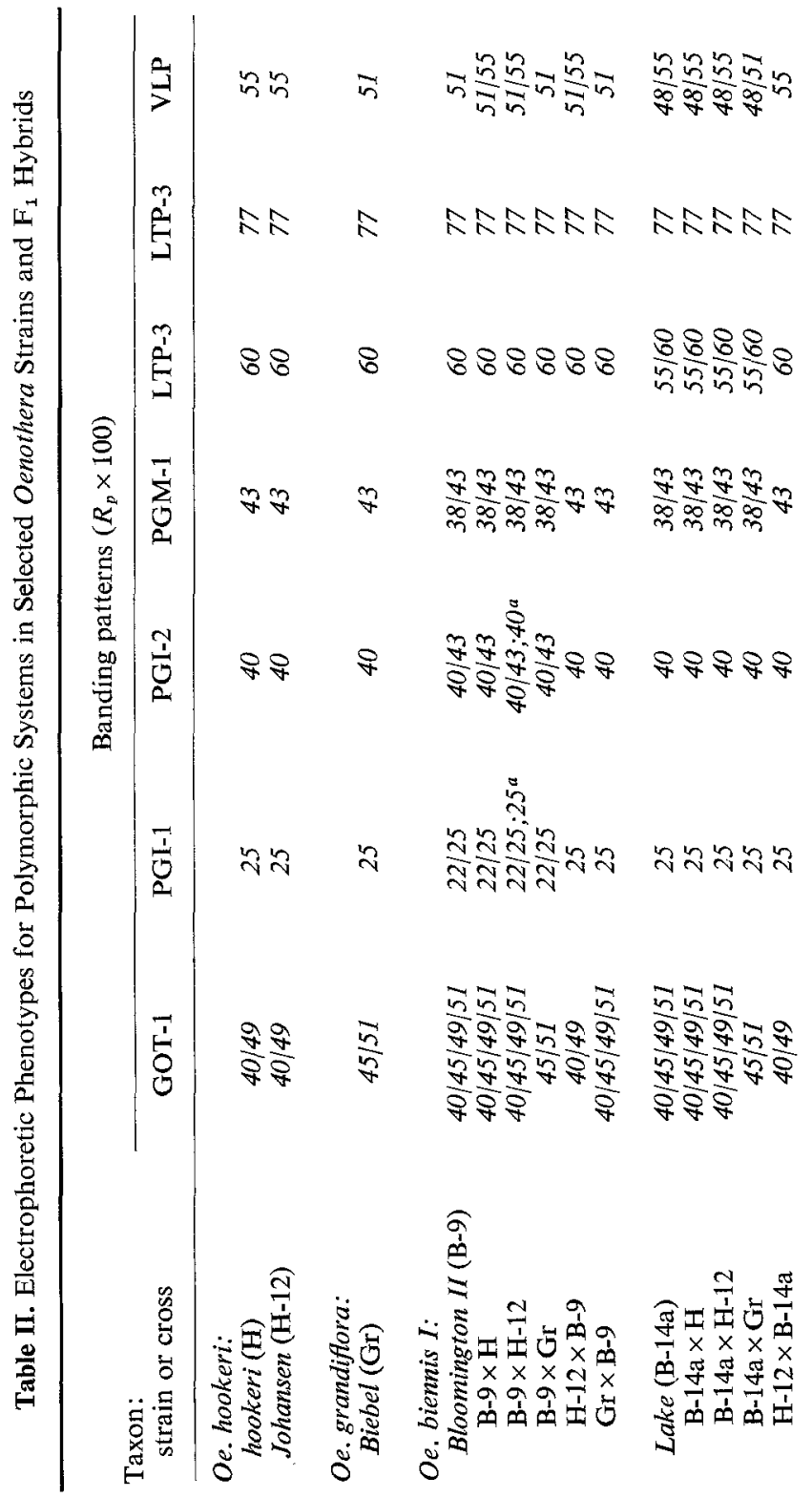




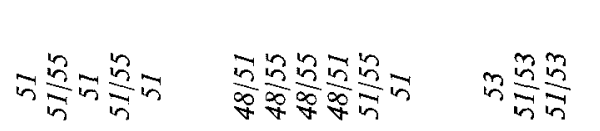

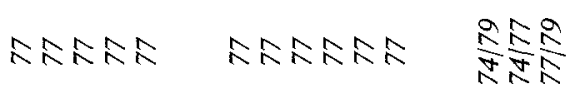

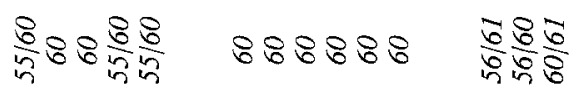

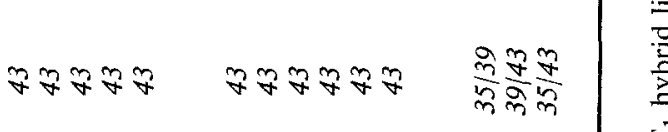

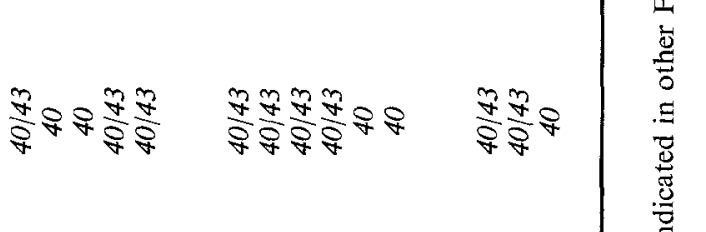

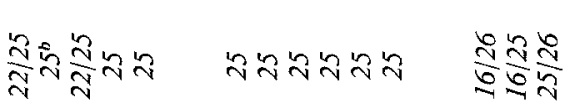

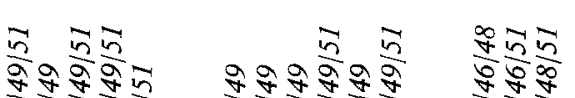

곱

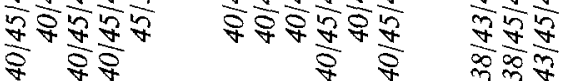

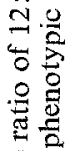

$8 \div$

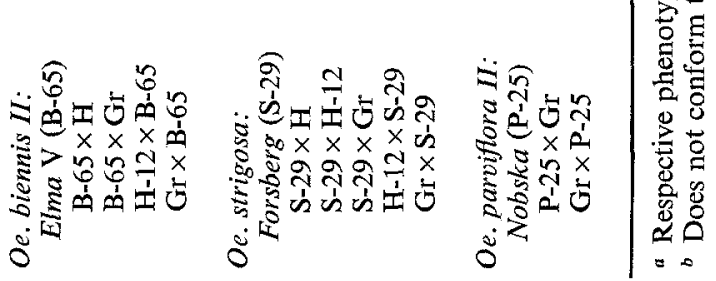


Bloomington II $\times$ Oe. hookeri $(40 / 45 / 49 / 51 \times 40 / 49)$ produces only $40 / 45 / 49 / 51$ progeny. The reciprocal cross produces only $40 / 49$ progeny. Bloomington $I I$ $\times$ Oe grandiflora $(40 / 45 / 49 / 51 \times 45 / 51)$ produces only $45 / 51$ progeny. The reciprocal cross produces only $40 / 45 / 49 / 51$ progeny. The preceding segregation patterns are consistent solely with exclusive transmission of the 45/51 doublet via Bloomington II ovules and the $40 / 49$ doublet via Bloomington II pollen. The uniformity within hybrid lines and the segregation of polymorphic systems solely among reciprocal crosses are consistent with the general absence of independent assortment among $\alpha$ and $\beta$ chromosome complexes and the predominant, exclusive transmission of complexes via opposite germ lines that characterizes permanent structural hybridity in Denothera.

Deviations from the predominant transmission patterns occur in some hybrids of ten of the 22 ring-forming strains (noted in Table IV). However, such deviations are manifest only in a few individuals of one or two $F_{1}$ lines involving each strain, and are concentrated in PGI systems. For example, the cross between a Bloomingion II ovule parent and the Johansen strain of Oe. hookeri (B-9 $\times \mathrm{H}-12$, Table II) produces a 12:2 ratio of phenotypes $22 / 25$ and 25 for PGI-1, and $40 / 43$ and 40 for PGI-2. The minority phenotypes are otherwise restricted to hybrid lines derived from a Bloomington II pollen parent. No segregants for either PGI system were observed in any other Bloomington II hybrids. Rare segregants of polymorphic PGI systems also occur in hybrids of four other ring-forming strains and of polymorphic VLP systems in hybrids of two additional ring-formers. Completely anomalous PGI transmission sequences are manifest in one cross of each of three ringformers. For example, the reciprocal crosses between the Oe. biennis II strain Elma V (B-65, Table II) and Oe. grandiflora testers and the Elma $V \times$ Johansen cross indicate that the 22 singlet of PGI-1 resides in Elma $V$ ovules. However, the Elma $V \times$ hookeri progeny exhibit only the 25 singlet and not the expected $22 / 25$ doublet. Other polymorphic systems segregate as expected in the last cross.

The frequency of segregants within hybrid lines derived from polymorphic ring-forming parents is within the normally observed range of recombination, $0.1-10.0 \%$ in hybrids of ring-forming oenotheras. Such rates tend to be higher in hybrid progeny than in the ring-forming strains themselves and restricted to genes positioned at the ends of chromosomes (for discussion, see Cleland, 1972, Chapter 10). However, in three instances of segregation within an $F_{1}$ line, we cannot distinguish between recombinational segregation and leakage in the gametophytic lethal or incompatibility systems. The frequency of such leakage, as judged from morphological segregation, is also enhanced in hybrid crosses (Cleland, 1972) and may be greater in the hybrid lines of the present study than indicated by electro- 
phoretic phenotypes (E. Steiner, unpublished). Nevertheless, the precise nature of both segregants within lines and anomalous transmission patterns remains to be resolved.

The $F_{1}$ phenotype data also demonstrate that for each staining zone (1) all crosses between parents with the same phenotype produce progeny with only that phenotype and (2) all crosses between parents with different singlet phenotypes (or different doublets at GOT-1) produce progeny with only complementary phenotypes. In combination with the inheritance patterns in polymorphic systems, these results indicate that electrophoretic variation within each polymorphic staining zone is encoded by codominant alleles at one locus. The absence of phenotypic segregation between multiple staining zones of GOT, PGI, PGM, and LTP also indicates that variation between zones is determined by separate loci. The encoding of enzyme morphs in each of the staining zones of the invariant systems ACP, ALP, and LGGP by separate loci may be inferred on bases of large mobility differences and differential rates of staining between morphs. This inference is further supported by the observation (Levin et al, 1972) of a rare doublet in ACP-1 which includes band 27 and exhibits no change in phenotype in ACP-2. The long-term selfing of the bivalent-forming tester strain reasonably insures that the nonsegregating doublets observed at G6PD and GOT-1 are encoded by homozygous genotypes. However, the present data do not resolve how many loci are responsible for such phenotypes. Preliminary results obtained from reelectrophoresis of some individual bands at GOT-1 suggest that the doublets may be mixtures of configurational isomers generated during electrophoretic procedures (M. Levy, unpublished). Tentatively, we will assume the most parsimonious explanation, single-locus determination.

Summarizing, we judge that, collectively, the electrophoretic variation resolved in the 11 enzyme systems assayed is under the genetic control of 20 loci. Allozymes encoded by Acp-1, Acp-2, Alp-1, Alp-2, Alp-3, Got-2, Lap, Lggp-1, Lggp-2, Ltp-1, Ltp-2, Ltp-3, Mdh, Pgi-1, Pgi-2, Pgm-1, Pgm-2, and $V l p$ occur as single bands and those at G6pd and Got-1, ostensibly, are manifest as nonsegregating doublets. All allozymes resolved are expressed codominantly, and heterozygotes (when present) exhibit additive phenotypes. Allelic designations which encode each of the allozymes observed in the study are summarized in Table III.

\section{Allelic Characterization of Chromosome Complexes}

The genotypes and the distribution of allozyme alleles at polymorphic loci among the chromosome complexes of the 22 ring-forming strains are presented in Table IV. Generally, within each species there are only two alleles per 
Table III. Allozyme Genotypes in Oenothera

\begin{tabular}{|c|c|c|}
\hline Locus & Allele & $\begin{array}{l}\text { Phenotype } \\
\left(R_{p} \times 100\right)\end{array}$ \\
\hline Acp-1 & $b$ & 27 \\
\hline Acp-2 & $b$ & 61 \\
\hline Alp-1 & $b$ & 30 \\
\hline \multirow[t]{2}{*}{ Alp -2} & $a$ & 60 \\
\hline & $b$ & 67 \\
\hline Alp -3 & $b$ & 93 \\
\hline G6pd & $b$ & $44 / 49$ \\
\hline \multirow[t]{4}{*}{ Got-1 } & $a$ & $40 / 49$ \\
\hline & $b$ & $45 / 51$ \\
\hline & $c$ & $38 / 46$ \\
\hline & $d$ & $43 / 48$ \\
\hline \multirow{2}{*}{ Got-2 } & $a$ & 57 \\
\hline & $b$ & 59 \\
\hline \multirow[t]{4}{*}{ Lap } & $a$ & 65 \\
\hline & $b$ & 60 \\
\hline & $c$ & 62 \\
\hline & $d$ & 64 \\
\hline Lggp-1 & $b$ & 50 \\
\hline Lggp-2 & $b$ & 65 \\
\hline Ltp-1 & $b$ & 33 \\
\hline \multirow[t]{4}{*}{ Ltp-2 } & $a$ & 55 \\
\hline & $b$ & 60 \\
\hline & $c$ & 56 \\
\hline & $d$ & 61 \\
\hline \multirow[t]{3}{*}{$L t p-3$} & $a$ & 77 \\
\hline & $b$ & 74 \\
\hline & $c$ & 79 \\
\hline$M d h$ & $b$ & 88 \\
\hline \multirow[t]{4}{*}{$P g i-1$} & $a$ & 22 \\
\hline & $b$ & 25 \\
\hline & $d$ & 16 \\
\hline & $e$ & 26 \\
\hline \multirow[t]{2}{*}{$P g i-2$} & $a$ & 40 \\
\hline & $b$ & 43 \\
\hline \multirow[t]{5}{*}{ Pgm-1 } & $a$ & 38 \\
\hline & $b$ & 43 \\
\hline & $c$ & 47 \\
\hline & $d$ & 35 \\
\hline & $e$ & 39 \\
\hline \multirow[t]{2}{*}{$P g m-2$} & $a$ & 57 \\
\hline & $b$ & 55 \\
\hline \multirow[t]{5}{*}{$V l p$} & $a$ & 48 \\
\hline & $b$ & 51 \\
\hline & $c$ & 55 \\
\hline & $d$ & 50 \\
\hline & $e$ & 53 \\
\hline
\end{tabular}


Table IV. Distribution of Alleles at Polymorphic Loci ${ }^{a}$ Among Chromosome Complexes in Oenothera

\begin{tabular}{|c|c|c|c|c|c|c|c|c|c|c|c|c|c|c|}
\hline \multirow{2}{*}{$\begin{array}{r}\text { Taxon: } \\
\text { strain }\end{array}$} & \multicolumn{2}{|c|}{ Got-1 } & \multicolumn{2}{|c|}{ Pgi-1 } & \multicolumn{2}{|c|}{ Pgi-2 } & \multicolumn{2}{|c|}{$P g m-1$} & \multicolumn{2}{|c|}{ Ltp-2 } & \multicolumn{2}{|c|}{$L t p-3$} & \multicolumn{2}{|c|}{$V l p$} \\
\hline & $\bar{\alpha}$ & $\bar{\beta}$ & $\bar{\alpha}$ & $\bar{\beta}$ & $\bar{\alpha}$ & $\bar{\beta}$ & $\bar{\alpha}$ & $\bar{\beta}$ & $\bar{\alpha}$ & $\bar{\beta}$ & $\bar{\alpha}$ & $\bar{\beta}$ & $\alpha$ & $\beta$ \\
\hline \multicolumn{15}{|l|}{ Oe. biennis I: } \\
\hline Baltimore & $b$ & $a$ & $b$ & $b$ & $b$ & $a$ & $b$ & $b$ & $b$ & $b$ & $a$ & $a$ & $a$ & $b$ \\
\hline Bestwater I & $a$ & $a$ & $b$ & $b$ & $a^{* *}$ & $b$ & $b$ & $b$ & $a$ & $b$ & $a$ & $a$ & $a$ & $b$ \\
\hline Bestwater II & $a$ & $a$ & $b$ & $b$ & $a$ & $a$ & $b$ & $b$ & $b$ & $b$ & $a$ & $a$ & $a$ & $b$ \\
\hline Birch Tree I & $b$ & $a$ & $b$ & $b$ & $b^{*}$ & $a^{*}$ & $a$ & $a$ & $b$ & $b$ & $a$ & $a$ & $a$ & $b$ \\
\hline Bloomington II & $b$ & $a$ & $a$ & $b$ & $b^{*}$ & $a^{*}$ & $a$ & $b$ & $b$ & $b$ & $a$ & $a$ & $b$ & $b$ \\
\hline Chicaginensis & $a$ & $a$ & $b$ & $b$ & $a$ & $a$ & $b$ & $b$ & $b$ & $b$ & $a$ & $a$ & $a^{*}$ & $c$ \\
\hline Coshocton I & $b$ & $b$ & $b$ & $b$ & $a$ & $b$ & $b$ & $b$ & $b$ & $b$ & $a$ & $a$ & $b$ & $b$ \\
\hline Hot Springs & $b$ & $b$ & $b$ & $b$ & $a$ & $a$ & $b$ & $b$ & $b$ & $b$ & $a$ & $a$ & $a$ & $b$ \\
\hline Lake & $b$ & $a$ & $b$ & $b$ & $a$ & $a$ & $a$ & $b$ & $a$ & $b$ & $a$ & $a$ & $a$ & $c$ \\
\hline
\end{tabular}

Oe. biennis $I I$ :

$\begin{array}{lllllllllllllll}\text { Buck Creek } & a & b & b & a & a^{* *} & b & b & b & b & b & a & a & b & a \\ \text { Corning II } & a & a & b^{*} & a & b^{*} & a^{*} & b & b & b & b & a & a & b & b \\ \text { Elma II } & a & b & b & b & a & a & b & b & b & a & a & a & b & b \\ \text { Elma V } & a & b & a^{* *} & b & a & b & b & b & b & a & a & a & b & b \\ \text { Tonawanda I } & a & b & b & b & a & a & b & b & b & b & a & a & c & c\end{array}$

Oe, strigosa:

$\begin{array}{lllllllllllllll}\text { Fargo } & a & a & b & b & a & a & b & b & b & b & a & a & b & b \\ \text { Forsherg } & a & a & b & b & b^{*} & a & b & b & b & b & a & a & a & b \\ \begin{array}{l}\text { Heber } \\ \text { N. Colorado }\end{array} & a & a & b & b & b & b & b & b & b & b & a & a & c^{*} & a \\ \quad \text { Springs } & a & a & b & b & b & a & b & b & b & b & a & a & b & b\end{array}$

Oe. parviflora:

$\begin{array}{lllllllllllllll}\text { Angustissima } & d & d & e & e & a & b & e & e & c & d & b & c & d & b \\ \text { Eriensis } & d & c & e^{*} & d^{*} & a & a & e & e & c & d & c & c & b & b \\ \text { Iron Mountain } & d & c & e & e & a & a & d & e & d & d & c & c & e & e \\ \text { Nobska } & c & d & d & e & b & a & d & e & c & d & b & c & e & e\end{array}$

a Not determined for Lap in Oe. parviflora.

* Minor $(\leqslant 10 \%)$ variation in transmission.

** Anomalous transmission in some crosses with Oe. hookeri.

polymorphic locus. Despite this meager allelic array, each of the strains is genotypically unique, and a total of 28 different genotypes are manifest among the 44 chromosome complexes characterized.

Most of the genotypic heterogeneity is concentrated among the "biennis" genomes of Oe. biennis ( $\alpha$-biennis $I$ and $\beta$-biennis $I I$ ) and in Oe. parviflora complexes. Among the nine $\alpha$-biennis $I$ complexes, only two bear common allelic constitutions. Differences between the complexes range from zero to six alleles, with a majority of comparisons within the group differing by two or three alleles. The genotypes of the five $\beta$-biennis $I I$ complexes are distinctive both inter se and in comparison with the $\alpha$-biennis $I$ complexes. Within the $\beta$-biennis II complexes, allelic differences range from one to four alleles, 
with a majority of comparisons differing by three or four alleles. Each of the $O e$. parviflora complexes exhibits a different genotype, and, by virtue of unique alleles at most loci, all are distinct from genotypes in the other ringforming taxa. The least genotypic heterogeneity is found among "strigosa" genomes. At polymorphic loci, a genotype containing Got-1 ${ }^{a}$, Pgi-1 ${ }^{b}$, Pgi-2 ${ }^{a}$, $P g m-I^{b}, L t p-2^{b}$, and $V l p^{b}$ is present in nine of the $22(41 \%) \beta$-biennis $I$, $\alpha$-biennis $I I$, and $\alpha$ - and $\beta$-strigosa complexes. All but two of the remaining strigosa complexes differ by only one or two alleles.

As a corollary to the differential heterogeneity among various genomes, striking differences in allele frequency are suggested between $\alpha$ and $\beta$ complexes of each group of Oe. biennis and between those of Oe. parviflora (Table V). Differences are most dramatic at Got-1, Ltp-2, and Vlp in Oe. biennis and at Ltp-2 and Pgm-1 in Oe. parviflora. Moreover, such "linkage disequilibria" in Oe. biennis include all polymorphic loci and are correlated

Table V. Allele Frequencies ( $\%$ ) at Major Polymorphic Loci in Complexes of Analyzed Ring-Forming Strains of Oenothera

\begin{tabular}{|c|c|c|c|c|c|c|c|c|c|}
\hline \multirow[b]{2}{*}{ Locus } & \multirow[b]{2}{*}{ Allele } & \multicolumn{2}{|c|}{ biennis I } & \multicolumn{2}{|c|}{ biennis II } & \multicolumn{2}{|c|}{ strigosa } & \multicolumn{2}{|c|}{ parviflora } \\
\hline & & $\alpha$ & $\beta$ & $\alpha$ & $\beta$ & $\alpha$ & $\beta$ & $\alpha$ & $\beta$ \\
\hline \multirow[t]{4}{*}{ Got-1 } & $a$ & 33 & 78 & 100 & 20 & 100 & 100 & $\mathbf{0}$ & 0 \\
\hline & $b$ & 66 & 22 & 0 & 80 & 0 & 0 & 0 & 0 \\
\hline & $c$ & 0 & 0 & 0 & 0 & 0 & 0 & 25 & 50 \\
\hline & $d$ & 0 & 0 & 0 & 0 & 0 & 0 & 75 & 50 \\
\hline \multirow[t]{4}{*}{$L p t-2$} & $a$ & 22 & 0 & 0 & 40 & 0 & 0 & 0 & 0 \\
\hline & $b$ & 78 & 100 & 100 & 60 & 100 & 100 & 0 & 0 \\
\hline & $c$ & 0 & 0 & 0 & 0 & 0 & 0 & 75 & 0 \\
\hline & $d$ & 0 & 0 & 0 & 0 & 0 & 0 & 25 & 100 \\
\hline \multirow[t]{4}{*}{$P g i-1$} & $a$ & 11 & 0 & 20 & 40 & 0 & 0 & 0 & 0 \\
\hline & $b$ & 88 & 100 & 80 & 60 & 100 & 100 & 0 & 0 \\
\hline & $d$ & 0 & 0 & 0 & 0 & 0 & 0 & 25 & 25 \\
\hline & $e$ & 0 & 0 & 0 & 0 & 0 & 0 & 75 & 75 \\
\hline \multirow[t]{2}{*}{$\mathrm{Pgi-2}$} & $a$ & 66 & 78 & 80 & 60 & 25 & 75 & 75 & 75 \\
\hline & $b$ & 33 & 22 & 20 & 40 & 75 & 25 & 25 & 25 \\
\hline \multirow[t]{4}{*}{$P g m-1$} & $a$ & 33 & 11 & 0 & 0 & 0 & 0 & 0 & 0 \\
\hline & $b$ & 66 & 88 & 100 & 100 & 100 & 100 & 0 & 0 \\
\hline & $d$ & 0 & 0 & 0 & 0 & 0 & 0 & 50 & 0 \\
\hline & $e$ & 0 & 0 & 0 & 0 & 0 & 0 & 50 & 100 \\
\hline \multirow[t]{5}{*}{$V l p$} & $a$ & 78 & 0 & 0 & 20 & 25 & 25 & 0 & 0 \\
\hline & $b$ & 22 & 78 & 100 & 60 & 50 & 75 & 25 & 50 \\
\hline & $c$ & 0 & 22 & 0 & 20 & 25 & 0 & 0 & 0 \\
\hline & $d$ & 0 & 0 & o & 0 & 0 & 0 & 25 & 0 \\
\hline & $e$ & 0 & 0 & 0 & 0 & 0 & 0 & 50 & 50 \\
\hline
\end{tabular}


with the genomic origin of complexes. Among the 14 Oe. biennis strains characterized, there are 34 heterozygous loci (Table IV). At 30 of these loci, the minority allele at each locus (Table V) is specific to the "biennis" genome; i.e., Got- $1^{b}, P g i-1^{a}, P g i-2^{b}, P g m-1^{a}, L t p-2^{a}$, and $V l p^{a}$ reside primarily in $\alpha$ biennis $I$ and $\beta$-biennis II complexes. The opposite situation occurs at $P g i-2$ in Coshocton I and Bestwater I and at Pgi-1 in Buck Creek and Elma V. Curiously, the latter three strains are those in which anomalous PGI transmission was detected. Intergenomic allele frequency differences also are present in Oe. parviflora, where the minority alleles Pgm- $I^{d}, L t p-2^{c}$, and $L t p-3^{b}$ are confined to $\alpha$ complexes (non-"parviflora" genomes). However, none of these alleles is present in other "biennis" or "strigosa" genomes.

Correlations between allozymic variation and the genomic origin of complexes do not extend to segmental arrangements within a group of complexes. For example, the segmental arrangement $1 \cdot 2,3 \cdot 4,5 \cdot 14,7 \cdot 10,9 \cdot 8$, $11 \cdot 12,13.6$ is manifest in 56 of the $78 \alpha$-biennis $I$ complexes fully analyzed by Cleland and associates and is judged to be the original $\alpha$ arrangement in the taxon (Cleland, 1972). Allozyme genotypes in this arrangement have been determined in five polymorphic strains, Baltimore, Md., Birch Tree I, Mo., Bloomington II, Ind., Hot Springs, Ark., and Lake, Va. (Table IV) and in seven uniformly homozygous strains, Hilltop, Ind., Walkerton, Ind., Cardiff Delta, Md., Cambridge I, Ohio, Lemoyne, Pa., Camp Perry, Va., and Petersburg, Va. (Levy and Levin, 1975, their Table 7). Ten genotypes are present in these 12 strains, with most differing by two or three alleles and Bestwater I and Bloomington II distinct inter se at all six polymorphic loci. Conversely, the greatest cytological diversity is found among complexes which are least variable genically, $\beta$-biennis $I, \alpha$-biennis $I I$, and both complexes of $O e$. strigosa. Further, it also is apparent from the above homozygous strains that complexes of different origins and completely different segmental arrangements may share the same allozyme genotypes. This phenomenon is also manifest in polymorphic strains, as evidenced by the common genotypes of $\alpha$-Elma $V$ and $\beta$-Corning II (Table IV).

\section{CONCLUSIONS}

Allozymes in the structural heterozygotes of Oenothera are inherited as single-locus, codominant markers. However, permanent translocation heterozygosity virtually eliminates the usual independent assortment or recombination of allozyme variants. Within a polymorphic ring-forming strain, an allozyme variant is specific to only one of the seven-chromosome linkage groups, transmitted nearly exclusively through only one germ line, and destined generally for permanent residence in the parental genotype. New allozyme genotypes may arise solely via the infrequent processes of 
recombination, hybridization, or mutation. Within taxa, the distribution of allozyme variants among $\alpha$ and $\beta$ chromosome complexes is nonrandom, and apparent linkage disequilibria for allozyme alleles are correlated with differences in the genomic origin of complexes. However, there is no obvious relationship between allozyme genotype and chromosome segmental arrangement.

\section{REFERENCES}

Cleland, R. E. (1972). Oenothera: Cytogenetics and Evolution, Academic Press, New York. Levin, D. A. (1975). Genic heterozygosity and protein polymorphism among local populations of Oenothera biennis. Genetics 79:477.

Levin, D. A., Howland, G. P., and Steiner, E. (1972). Protein polymorphism and genic heterozygosity in a population of the permanent translocation heterozygote, Oenothera biennis. Proc. Natl. Acad. Sci. 69:1475.

Levy, M., and Levin, D. A. (1975). Genic heterozygosity and variation in permanent translocation heterozygotes of the Oenothera biennis complex. Genetics 79:493. 